\title{
MicroRNA-182 inhibits proliferation through targeting oncogenic ANUBL1 in gastric cancer
}

\author{
LIN TANG $^{1}$, FENG CHEN $^{2}$, ER-JUN PANG ${ }^{2}$, ZHI-QI ZHANG ${ }^{2}$, BING-WEI JIN ${ }^{2}$ and WEI-FENG DONG ${ }^{2}$ \\ ${ }^{1}$ School of Basic Medical Sciences, Nanjing University of Chinese Medicine, Nanjing 210023; ${ }^{2}$ Division of General Surgery, \\ Shanghai Jiao Tong University Affiliated Sixth People's Hospital, Shanghai 200233, P.R. China
}

Received November 3, 2014; Accepted December 22, 2014

DOI: $10.3892 /$ or.2015.3798

\begin{abstract}
MicroRNA-182 (miR-182) is significantly downregulated in human gastric tissue samples. Overexpression of miR-182 suppresses the proliferation and colony formation of gastric cancer cells. However, new aspects of the mechanism are still emerging in gastric cancer. ANUBL1, also known as ZFAND4 (zinc finger, AN1-type domain 4), its roles are scarely reported in cancer. In this study, we not only showed that ANUBL1 as an oncogene was upregulated and could promote proliferation of SGC-7901 cells, but also demonstrated that its overexpression led to a strong decrease of miR-182 expression and expression of $A N U B L 1$ was in turn directly downregulated by miR-182, thereby establishing a negative feedback loop between miR-182 and ANUBL1. The elucidation of the mechanisms of miR-182 targeting ANUBL1 in gastric cancer helps us to further understand the mechanism of gastric cancer initiation and progression.
\end{abstract}

\section{Introduction}

Gastric cancer (GC) causes $12 \%$ of all cancer-related deaths each year $(1,2)$. The interaction of multiple factors contributes to the development of gastric carcinogenesis, including regulatory changes in oncogenes and tumor suppressor genes $(3,4)$. The regulation of oncogenes and tumor suppressor genes occurs not only at the transcriptional level, but also at the posttranscriptional level.

MicroRNAs (miRNAs, miRs) are a new class of small ( 22 nucleotide) noncoding RNAs that negatively regulate protein-coding gene expression by targeting mRNA degradation or translation inhibition (5-7). Profiling studies revealed the contribution of aberrant miRNA expression to GC initiation and progression by perturbing the function of target genes $(8,9)$. Previous studies had shown that miR-182 is poorly expressed in human breast cancer stem cells, human and mouse normal

Correspondence to: Dr Feng Chen, Division of General Surgery, Shanghai Jiao Tong University Affiliated Sixth People's Hospital, Shanghai 200233, P.R. China

E-mail: chenfengmail@163.com

Key words: gastric cancer, ANUBL1, miR-182, proliferation mammary stem cells, and embryonic carcinoma (10). miR-182 could deregulate RGS17 by targeting its 3'UTR to ultimately suppress the occurrence of lung cancer (11). Although miR-182 is downregulated and can suppress cell growth and target cAMP-responsive element-binding protein 1 in human gastric cancer (12), the various mechanisms of miR-182 inhibiting proliferation of gastric cancer cells are still emerging.

The roles of ANUBL1, also known as ZFAND4, are scarely reported in cancer. Firstly, we demonstrated that ANUBL1 expression was specifically upregulated in gastric cancer and it was positively associated with grades of gastric cancer. Moreover, it promoted proliferation of gastric cancer SGC-7901 cells. Secondly, we analyzed the mechanism of ANUBL1 upregulation in gastric cancer. We predicted that miR-182 could target 3'UTR of ANUBL1 mRNA by bioinformatic methods and the following studies confirmed the prediction that miR-182 suppressed expression of ANUBL1 through targeting its 3'UTR. Moreover, we found that ANUBL1 could downregulate anti-proliferation-associated miRNA (miR-148b, miR-375 and miR-182) expression in SGC-7901 cells. Finally, we demonstrated that introduction of ANUBL1 cDNA lacking predicted sites of 3'-UTR abrogates miR-182 cellular function (unpublished data).

In this study, we not only showed that ANUBL1 as an oncogene was upregulated and could promote proliferation of SGC-7901 cells, but also demonstrated that its overexpression led to a strong decrease of miR-182 expression and expression of $A N U B L 1$ was in turn directly downregulated by miR-182, thereby establishing a negative feedback loop between miR-182 and ANUBL1. The elucidation of the mechanisms of miR-182 targeting ANUBL1 in gastric cancer helps us to further understand the mechanism of gastric cancer initiation and progression.

\section{Material and methods}

Human tissue samples. One hundred and fifty pairs of human gastric tissue samples were obtained from patients who underwent surgical resection at the Division of General Surgery, Shanghai Jiao Tong University Affiliated Sixth People's Hospital between 2009 and 2014 and were diagnosed with gastric cancer based on histopathological evaluation (32, WHO grade I; 34, WHO grade I; 40, WHO grade III; and 37, WHO grade IV). The mean age was 55 years (range: $30-74$ ). 
The matched non-tumor adjacent tissue was obtained from a segment of the resected specimens that was the farthest from the tumor $(>5 \mathrm{~cm})$. The samples were snap-frozen in liquid nitrogen and stored at $-80^{\circ} \mathrm{C}$. No local or systemic treatment was conducted in these patients before the operation. The use of human tissue samples followed internationally recognized guidelines as well as local and national regulations. Research carried out on humans followed international and national regulations. Medical ethics committees of Shanghai Jiao Tong University Affiliated Sixth People's Hospital approved the experiments undertaken. Informed consent was obtained from each individual.

Immunohistochemistry. Immunohistochemisty was performed using standard techniques. Antigen retrieval was performed by autoclaving. Incubation with $10 \%$ normal goat serum in phosphate-buffered saline was performed for $15 \mathrm{~min}$ to eliminate non-specific staining. Incubation with anti-ANUBL1 antibody (Abcam, Cambridge, MA, USA) was carried out. Finally, sections were lightly counterstained with $10 \%$ Mayer hematoxylin, dehydrated, mounted, and observed. Staining was evaluated by a pathologist and an investigator blinded to diagnosis. Sections were classified as - (negative), + (focal and weak immunoreactivity), ++ (diffuse and weak or focal and intense immunoreactivity), +++ (diffuse and intense immunoreactivity). The data were analyzed by SPSS 11.5 statistical package. Expression of ANUBL1 was evaluated according to the ratio of positive cells per specimen and staining intensity (13). The comparison of the expression rates was by Chi-square test.

Cell culture, plasmids and transfection. Human gastric cancer SGC-7901 cells were obtained from MD Anderson Cancer Center (Houston, TX, USA). SGC-7901 human gastric cancer cells were maintained in RPMI-1640 (Gibco, Grand Island, NY, USA) with $10 \%$ heat-inactivated fetal bovine serum (Gibco), $100 \mathrm{IU} / \mathrm{ml}$ penicillin, and $0.1 \mathrm{mg} / \mathrm{ml}$ streptomycin, in a humidified $5 \%$ atmosphere of $\mathrm{CO}_{2}$ at $37^{\circ} \mathrm{C}$. ANUBL1-expressing plasmids/empty vector (pcDNA3.1) and sh-ANUBL1/scramble were purchased from the National RNAi Core Facility in Academic Sinica, Taipei, Taiwan. The ANUBL1-expressing plasmids/empty vector (pcDNA3.1) and sh-ANUBL1/scramble used for each transfection is $10 \mathrm{nM}$, unless otherwise specified. Pre-miR-182 and control-miR were purchased from Ambion (Austin, TX, USA). For transfection experiments, the cells were cultured in serum-free medium without antibiotics at $60 \%$ confluence for $24 \mathrm{~h}$, and then transfected with transfection reagent (Lipofectamine 2000, Invitrogen, Carlsbad, CA, USA) according to manufacturer's instructions. After incubation for $6 \mathrm{~h}$, the medium was removed and replaced with normal culture medium for $48 \mathrm{~h}$.

Colony formation. For colony formation assay, cells were transfected with ANUBL1 expressing plasmids or sh-ANUBL1 plasmids for $24 \mathrm{~h}$, and then seeded in a 6-well plate. FBS $(0.2 \mathrm{ml})$ was added per well on day 5. After 9-10 days of incubation, plates were washed with PBS and stained with $0.1 \%$ of crystal violet. Colonies with over 50 cells were manually counted. Plating efficiency was calculated by dividing the number of colonies formed in the treated group by that in control.
Cell-cycle analysis. Cells $\left(8.0 \times 10^{5}\right)$ were seeded into a $100-\mathrm{mm}$ culture plate and allowed to attach overnight. The cells were transfected with plasmids for $24 \mathrm{~h}$, washed twice with $\mathrm{NaCl} / \mathrm{Pi}$, and then centrifuged at $200 \mathrm{x} \mathrm{g}$ at room temperature. The pellet was resuspended in $1 \mathrm{ml}$ cold $\mathrm{NaCl} / \mathrm{Pi}$ and fixed in $70 \%$ ethanol for at least $12 \mathrm{~h}$ at $4^{\circ} \mathrm{C}$. The fixed cells were incubated with $100 \mu \mathrm{l}$ DNase-free RNaseA $(200 \mu \mathrm{g} / \mathrm{ml})$ for $30 \mathrm{~min}$ at $37^{\circ} \mathrm{C}$, and then $1 \mathrm{mg} / \mathrm{ml}$ propidium iodide was added. The stained cells were analyzed using a fluorescence-activated cell sorter (BD Accuri C6; BD Biosciences, Ann Arbor, MI, USA). The percentages of cells in the G1, S and G2/M phases of the cell cycle were determined using CellQuest Pro software (FlowJo, Ashland, OR, USA).

MTT assay. The effect of the cell proliferation was assessed by 3-(4, 5-dimethylthiazol-2-yl)-2, 5-diphenyltetrazolium (MTT; Sigma, St. Louis, MO, USA) assay. SGC-7901 cells were transfected with ANUBL1 or pcDNA3.1 (mock), sh-ANUBL1 or scramble (mock). These cells were incubated in a humidified atmosphere with $5 \% \mathrm{CO}_{2}$. Then, MTT solution $(4.14 \mathrm{mg} / \mathrm{ml})$ was added to each well and the optical density was measured after incubation at $37^{\circ} \mathrm{C}$ for $4 \mathrm{~h}$ (14). Absorbance was directly proportional to the number of survival cells.

Bromodeoxyuridine labeling and immunofluorescence. Cells grown on coverslips (Fisher, Pittsburgh, PA) were incubated with bromodeoxyuridine (BrdUrd) for $1 \mathrm{~h}$ and stained with anti-BrdUrd antibody (Upstate Biotechnology, Temecula, CA, USA) according to the manufacturer's instruction. Images were acquired under a laser scanning microscope (Axioskop 2 plus, Carl Zeiss Co. Ltd., Jena, Germany).

Reverse transcription-polymerase chain reaction and realtime for ANUBL1. Total RNA was isolated from cells or tissues using Trizol reagent (cat no. 11647229001, Invitrogen). cDNA was synthesized from $1 \mu \mathrm{g}$ of total RNA in a $20 \mu \mathrm{l}$ reverse transcription (RT) system followed by PCR amplification in a $50 \mu \mathrm{l}$ PCR system performed using an RT-PCR kit (cat no. A3500, Promega, Madison, WI, USA). Housekeeping gene Glyceraldehyde-3-phosphate dehydrogenase (GAPDH) was used as RNA loading control. The PCR primer sequences are as follows: GAPDH: Forward - ATTCAACGGCACAGT CAAGG, Reverse - GCAGAAGGGGCGGAGATGA; PCNA: Forward - CTGTAGCGGCGTTGTTGC, Reverse - TCG TTGATGAGGTCCTTG; Ki67: Forward - CAACTATCCT CGTCTGTCC, Reverse - GGTCCCTAAAGATGTGCT; p21: Forward - CCCGTGAGCGATGGAACT, Reverse - CGA GGCACAAGGGTACAAGA; p53: Forward - CCTCCTCA GCATCTTATCCG, Reverse - CACAAACACGCACCTC AAA; Rb: Forward - AAGGTTTCAGGGTATCAG, Reverse GTGGGTCTGTATGTTGTG; E2F1: Forward - AAGAAC CGCTGTTGTCCCG, Reverse - TCGAGGCCGAAGTGG TAGTC; CDK2: Forward - AGAAACAAGTTGACGGGAG, Reverse - GAAGAGGAATGCCAGTGAG; CDK4: Forward CAGTTCGTGAGGTGGCTTTA, Reverse - GGGGTGCC TTGTCCAGATA; CDK6: Forward - TGCCCACTGAAA CCATAAAGG, Reverse - ATCCACAGCGTGACGACCA; The PCR cycles were conducted according to manufacturer's instruction and the PCR products were analyzed by agarose gel electrophoresis. Gels were photographed and densities of 
the bands were determined with a computerized image analysis system (Alpha Innotech, San Leandro, CA, USA). The area of each band was calculated as the integrated density value (IDV). Real-time PCR for ANUBL1 was done with a Power SYBR Green PCR Master Mix (Applied Biosystems, Carlsbad, CA, USA) according to the manufacturer's protocol. The primer sequences for ANUBL1: Forward - CCGAGACAGATA ACGAGGAT-3', Reverse - GAGGTCTTTCTGCTAGAC-3'.

Western blot analysis. Protein extracts from the cells or tissue samples were separated by SDS-PAGE, transferred to PVDF membrane (15). After incubation with rabbit anti-ANUBL1, anti-Ki67, anti-PCNA, anti-p21, anti-p53, anti-RB, anti-CDK2, anti-CDK4, anti-CDK6 (all from Abcam) and anti- $\beta$-actin antibodies overnight at $4^{\circ} \mathrm{C}$, IRDye $\mathrm{TM}^{\mathrm{TM}}-800$ conjugated antirabbit secondary antibodies (Li-COR, Biosciences, Lincoln, NE, USA) were used for $30 \mathrm{~min}$ at room temperature. The specific proteins were visualized by Odyssey ${ }^{\mathrm{TM}}$ Infrared Imaging System (Gene Company, Lincoln, NE, USA).

Immunofluorescence analyses. For immunofluorescence analyses, cells were plated on glass coverslips in six-well plates and transfected with $30 \mathrm{nM}$ pre-miR-182 or control miR. At $36 \mathrm{~h}$ after transfection, coverslips were stained with the mentioned anti-ANUBL1 antibodies. Alexa Fluor 488 goat anti-mouse IgG antibody or goat anti-rabbit IgG antibody was used as secondary antibody (Invitrogen). Coverslips were counterstained with DAPI (Invitrogen-Molecular Probes, Eugene, Oregon, USA) for visualization of the nuclei. Microscopic analysis was performed with a confocal laser-scanning microscope (Leica Microsystems, Bensheim, Germany). Fluorescence intensities were measured in a few viewing areas for 200-300 cells per coverslip and analyzed using ImageJ $1.37 \mathrm{v}$ software (http://rsb.info.nih.gov/ij/index.html).

miRNA microarray. Total RNA from cultured cells, with efficient recovery of small RNAs, was isolated using the mirVana miRNA Isolation kit (cat no. AM1561, Ambion). cRNA for each sample was synthesized by using 3'IVT Express kit according to the manufacturer's protocols. The purified cRNA was fragmented by incubation in fragmentation buffer (provided in the $3^{\prime} \mathrm{IVT}$ express kit) at $95^{\circ} \mathrm{C}$ for $35 \mathrm{~min}$ and chilled on ice. The fragmented labeled cRNA was applied to MicroRNA 2.0 Array and hybridized in Genechip hybridization oven 640 , at $45^{\circ} \mathrm{C}$ for $18 \mathrm{~h}$. After washing and staining in Genechip fluidics station 450 , the arrays were scanned by using Genechip scanner 3000 (all from Affymetrix, Santa Clara, CA, USA). The gene expressions levels of samples were normalized and compared by using Partek GS 6.5 (Partek, Inc, St. Louis, MO, USA). Average-linkage hierarchical clustering of the data was applied by using the Cluster and the results were obtained by using TreeView developed by Michael Eisen (Stanford University, CA, USA; http://rana. lbl.gov).

Real-time PCR for microRNAs. Detection of the mature form of miRNAs was performed using the mirVana qRT-PCR miRNA Detection kit (cat no. AM1558, Ambion), according to the manufacturer's instructions. The U6 small nuclear RNA was used as an internal control.
Methods of bioinformatics. The analysis of potential microRNA target sites using 4 commonly used prediction algorithms - miRanda (http://www.microrna.org/), TargetScan (http://www.targetscan.org), miRDB (http://mirdb.org/ miRDB/) and PicTar (http://pictar.bio.nyu.edu).

Luciferase reporter assay. The 3'UTR of ANUBL1 was donated by Yao Xu (Harvard University, USA) Briefly, it was amplified using cDNA from SGC-7901 cells, cloned into pRL-TK (Promega), checked for orientation, sequenced and named Luc-ANUBL1-WT. Site-directed mutagenesis of the miR-182 target-site in the ANUBL1-3'-UTR was carried out using Quik change mutagenesis kit (cat no. 200519, Stratagene, Heidelberg, Germany), with Luc-ANUBL1-WT as a template. For reporter assays, SGC-7901 cells were transiently transfected with wt or mutant reporter plasmid and pre-miR-182 (as indicated in Fig.4) using Lipofectamine 2000 (Invitrogen). Reporter assays were performed $36 \mathrm{~h}$ post-transfection using the Dual-luciferase assay-system (Promega), normalized for transfection efficiency by cotransfected Renilla-luciferase.

\section{Results}

Aberrant ANUBL1 expression in gastric cancer. To assess the expression of ANUBL1 in gastric cancer, real-time PCR and western blot analysis were conducted in 7 pairs of gastric cancer tissues and matched adjacent normal tissue samples. The expression of ANUBL1 was consistently higher in the gastric cancer tissues than in normal tissues (Fig. 1A). These data support the notion that ANUBL1 may act as an oncogene in gastric cancer.

To show the general importance of ANUBL1 in pathogenesis of gastric cancer, we applied immunohistochemistry analyses to detect ANUBL1 expression in human gastric cancer specimens. Staining was evaluated by a pathologist and an investigator blinded to diagnosis. Sections of gastric cancer of every grade were divided into two groups $(-/+$ and $++/+++)$ (Fig. 1B). The expression rates of the ANUBL1 protein in $++/++$ group in gastric cancer of grades I, II, III and IV were 12.5, 29.4, 45.0 and 70.3\%, respectively (Fig. 1B). Statistical analysis showed that ANUBL1 expression level was higher in high-grade gastric cancer tissues than in low-grade ones, grades I vs. IV ( $p<0.001)$, grade II vs. grade IV $(p<0.001)$, and grade I vs. grade III $(\mathrm{p}<0.001)$.

In order to further confirm that high expression of ANUBL1 was associated with high-grade human gastric cancer, quantitative image analysis was performed to analyze ANUBL1 protein expression in these tissue sections and we found that ANUBL1 expression was indeed associated with grades of gastric cancer (Fig. 1C). These data implied that ANUBL1 as an oncogene was upregulated in gastric cancer tissues and associated with progression of gastric cancer.

ANUBL1 promotes proliferation of gastric cancer SGC-7901 cells. To investigate whether ANUBL1 contributes to proliferation of gastric cancer cells, firstly using western blot, we tested whether ANUBL1 expressing plasmids could stably express ANUBL1 protein in SGC-7901 cells. The results showed that ANUBL1 protein could be significantly increased by ANUBL1 expressing plasmids in the cells (Fig. 2A). 

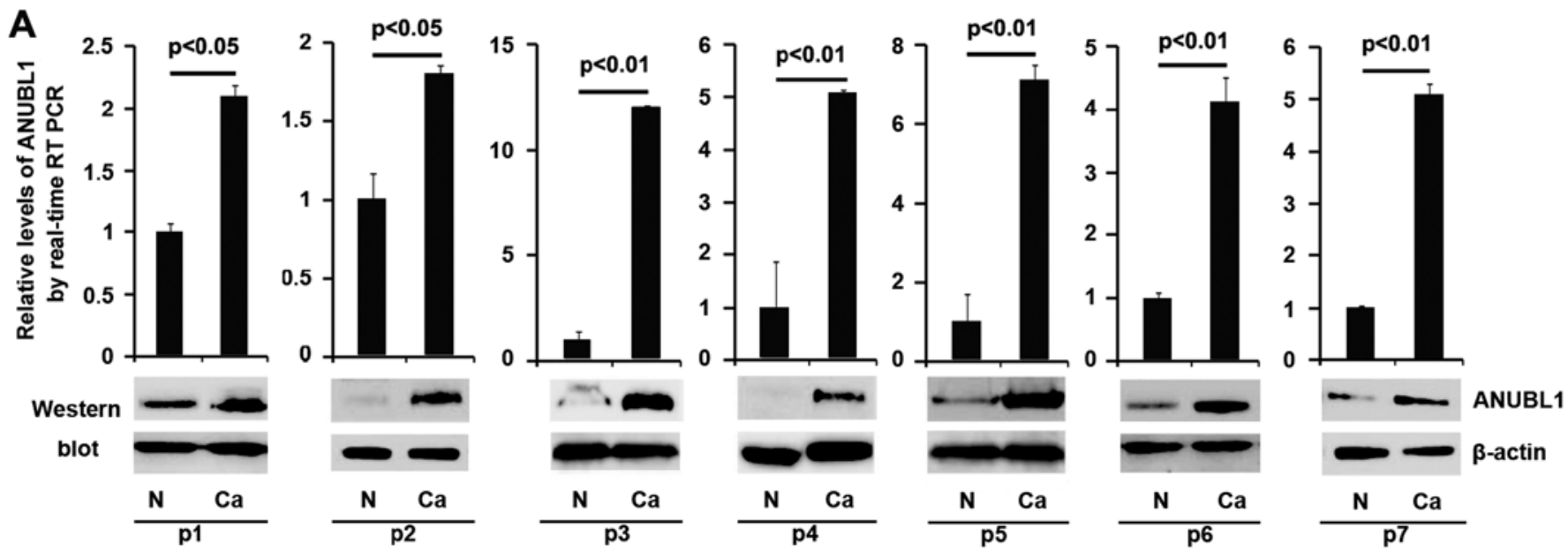

B

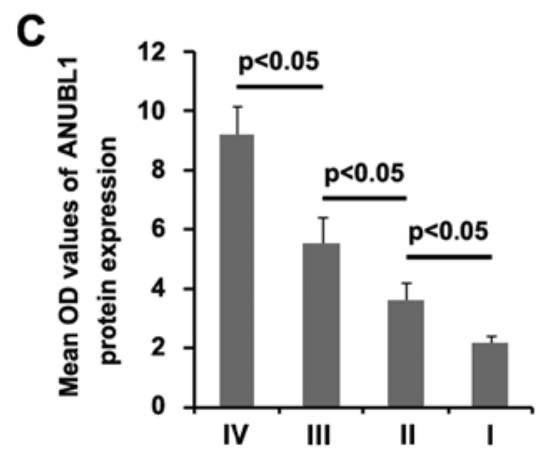

Figure 1. Aberrant ANUBL1 expression in gastric cancer. (A) Differential expression of ANUBL1 in 7 pairs of human gastric cancer tissues (Ca) and corresponding adjacent normal tissues $(\mathrm{N})$ were detected with real-time PCR and western blotting. U6 small nuclear RNA and $\beta$-actin was regarded as endogenously normalized $(\mathrm{n}=7)$. (B) The expression percentage of the ANUBL1 protein (immunohistochemistry) was classified as $-/+$ or $++/+++$, respectively, in gastric cancer diagnosed as grades I, II, III and IV ( $\mathrm{n}=143$ ). (C) Quantitative image analysis for ANUBL1. Mean OD values for ANUBL1 protein expression of immunohistochemical images in gastric cancer diagnosed as grades I, II, III and IV ( $n=143)$.

In order to identify the effect of ANUBL1 on colony formation, we performed colony formation assay. The results showed that over-expression of ANUBL1 significantly increased colony formation rate of SGC-7901 cells after transfection (Fig. 2B).

In addition, we also performed MTT assay to detect proliferation of SGC-7901 cells transfected with ANUBL1 expressing plasmids. The results showed that ANUBL1 promoted proliferation in SGC-7901 cells after $24 \mathrm{~h}$ of transfection and the promotion was dose- and time-dependent (Fig. 2C). To further show the effects of ANUBL1 on proliferation, we performed cell cycle analysis to analyze its effects on the cell cycle. The results showed higher $S$ phase fractions in SGC-7901 cells transfected with ANUBL1 than in SGC-7901 cells transfected with empty vector (Fig. 2D). To further identify that DNA synthesis promotion contributed to the higher S phase fractions in SGC-7901 cells transfected with ANUBL1, we performed Brdu incorporation assay to detect DNA synthesis in the cells. The results confirmed that ANUBL1 significantly promoted DNA synthesis in the cells and representative micrographs (left) and quantification (right) of BrdU incorporating-cells after transfection with ANUBL1 or empty vector (mock) are shown in Fig. 2E. In the following studies, we performed RT-PCR to identify whether mRNA of the proliferation markers were also affected by ANUBL1 in the cells. The results of RT-PCR showed that expression of PCNA, Ki67, CDK2, CDK4, CDK6, E2F1 was upregulated, and expression of $\mathrm{p} 21$ and $\mathrm{p} 53$ was downregulated by ANUBL1 in the cells (Fig. 2F). In addition, we performed western blot to further confirm that ANUBL1 could regulate these markers. The results of western blot demonstrated that PCNA, Ki67, CDK2, CDK4 and CDK6 expression were upregulated and p21 and p53 expression were downregulated by ANUBL1 (Fig. 2G). Due to lack of the E2F1 antibody, we did not analyze its protein expression in the cells. These data implied that ANUBL1 was upregulated and could promote proliferation in SGC-7901 cells.

Silencing ANUBL1 inhibits proliferation in gastric cancer $S G C-7901$ cells. We demonstrated that ANUBL1 overexpression promoted proliferation in SGC-7901 cells. To provide further evidence that ANUBL1 were involved in proliferation of SGC-7901 cells, we studied the effects of the sh-ANUBL1, an inhibitor of ANUBL1. After stable transfection, ANUBL1 expression was detected by western blotting. The results showed that exogenous sh-ANUBL1 significantly downregulated ANUBL1 expression in SGC-7901 cells (Fig. 3A). Colony formation of SGC-7901 cells were tested by colony formation assay. Silencing ANUBL1 significantly suppressed colony formation rate of SGC-7901 cells (Fig. 3B). Moreover, we also performed MTT assay to detect proliferation of SGC-7901 cells with sh-ANUBL1 and scramble transfection. 
A
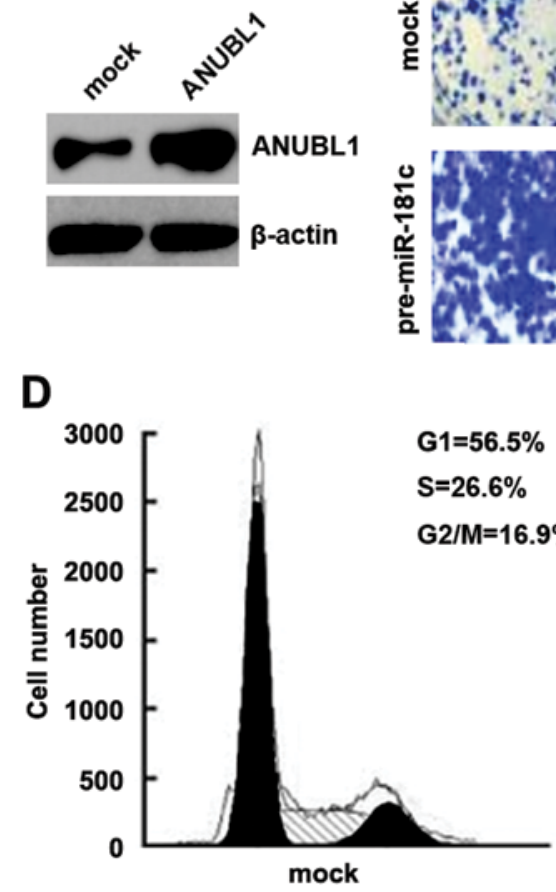

E

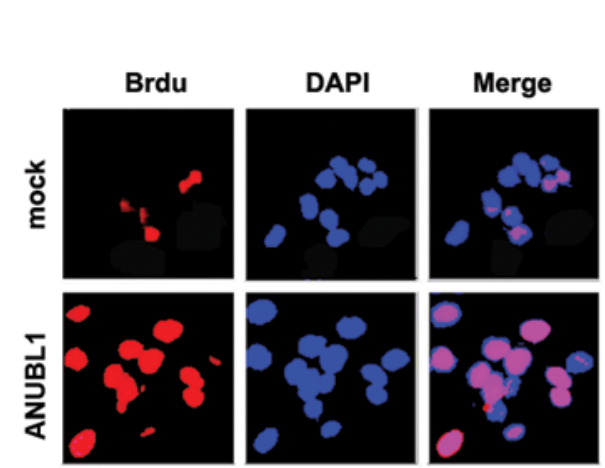

B

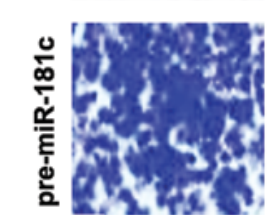

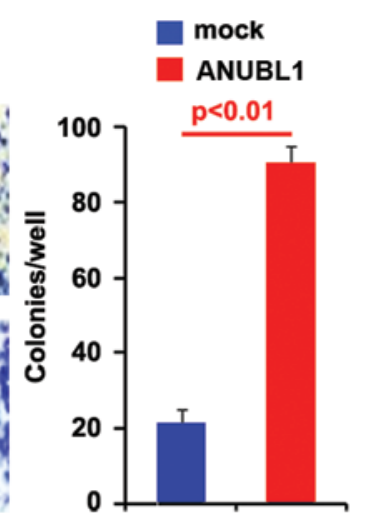

C

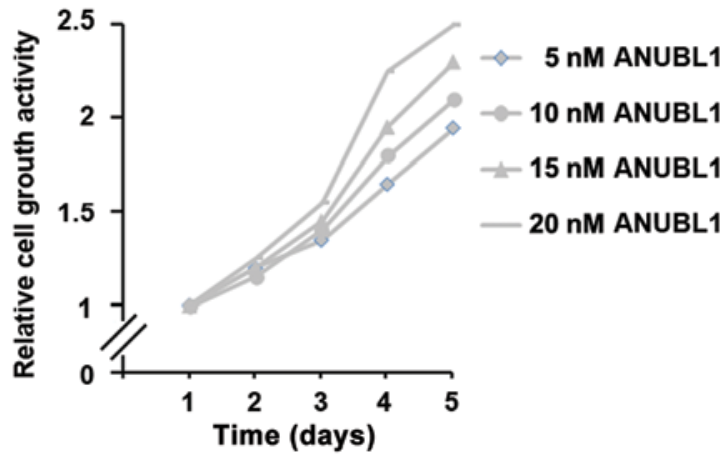

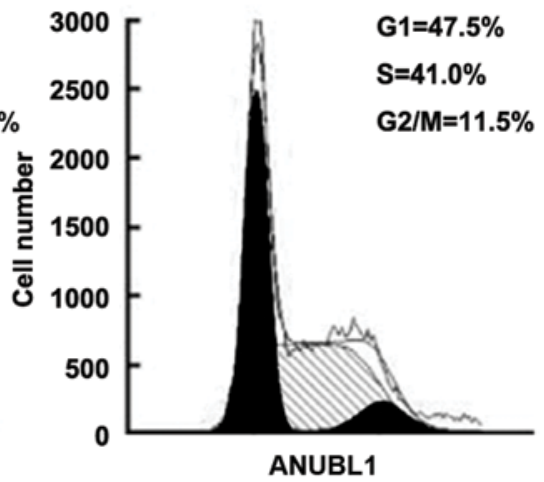

F

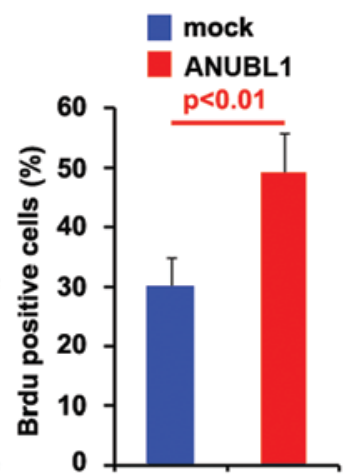

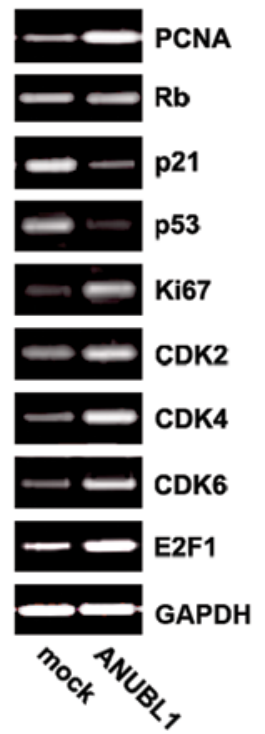

G

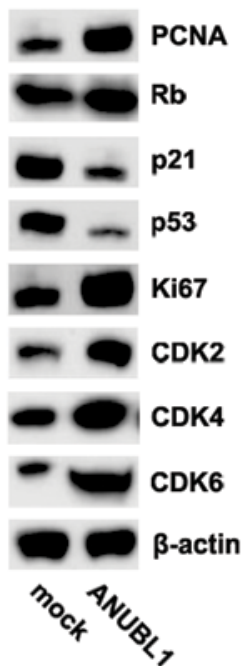

Figure 2. ANUBL1 promotes proliferation in gastric cancer SGC-7901 cells. (A) Western blot for ANUBL1 in SGC-7901 cells. SGC-7901 cells were transfected with ANUBL1 expressing plasmids or empty vector (mock). $\beta$-actin was a loading control (n=3). (B) Colony formation assay for SGC-7901 cells transfected with ANUBL1 expressing plasmids or empty vector (mock). Colonies with over 50 cells were counted. Representative micrographs (left) and quantification of colonies (right) after transfection with ANUBL1 expressing plasmids or empty vector (mock) (n=3). (C) MTT assay for SGC-7901 cells. SGC-7901 cells were transfected with ANUBL1 expressing plasmids as indicated and then cell viability was measured at the indicated time points by MTT assay ( $n=3)$. (D) Cell cycle analysis for SGC-7901 cells transfected with ANUBL1 or empty vector (mock). Histograms of DNA contents obtained by FACS analysis are shown. The percentages of each cell cycle stages are shown in the inset of the histograms ( $\mathrm{n}=3$ ). (E) Brdu incorporation assay for SGC-7901 cells. Representative micrographs (left) and quantification (right) of BrdU incorporating-cells after transfection with ANUBL1 expressing plasmids or empty vector (mock) (n=3). (F) RT-PCR for PCNA, Rb, p21, p53, Ki67, CDK2, CDK4, CDK6 and E2F1 in SGC-7901 cells infected with ANUBL1 or empty vector (mock). GAPDH was a loading control ( $\mathrm{n}=3)$. (G) Western blot for PCNA, Rb, p21, p53, Ki67, CDK2, CDK4 and CDK6 in SGC-7901 cells transfected with ANUBL1 or empty vector (mock). $\beta$-actin was a loading control $(n=3)$.

The results showed that sh-ANUBL1 inhibited proliferation in SGC-7901 cells, compared with scramble-transfected groups (Fig. 3C). To further show the effects of silencing ANUBL1 on proliferation, we performed cell cycle analysis to analyze the effect of sh-ANUBL1 on the cell cycle. The results showed that lower S phase fractions in SGC-7901 cells transfected with sh-ANUBL1 than in SGC-7901 cells transfected with scramble (Fig. 3D). To identify that DNA synthesis inhibition contributed to lower $S$ phase fractions in SGC-7901 cells transfected with sh-ANUBL1, we performed Brdu incorporation assay to detect DNA synthesis in the cells. The results confirmed that sh-ANUBL1 significantly suppressed DNA synthesis in the cells and representative micrographs and quantification of BrdU incorporating-cells after transfection with sh-ANUBL1 or scramble are shown in Fig. 3E. All the results confirmed that ANBUL1, as an oncogene promoted proliferation in SGC-7901 cells.

ANUBL1 is a target of miR-182 in gastric cancer SGC-7901 cells. Having demonstrated that ANUBL1 expression is specifically upregulated and is associated with progression of the disease and it promotes proliferation in SGC-7901 cells, 
A

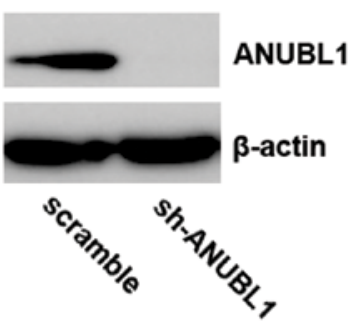

B

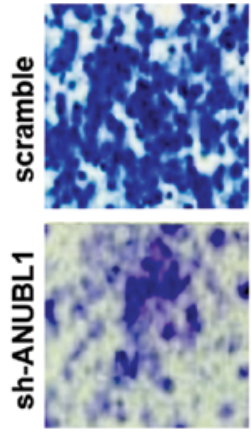

D

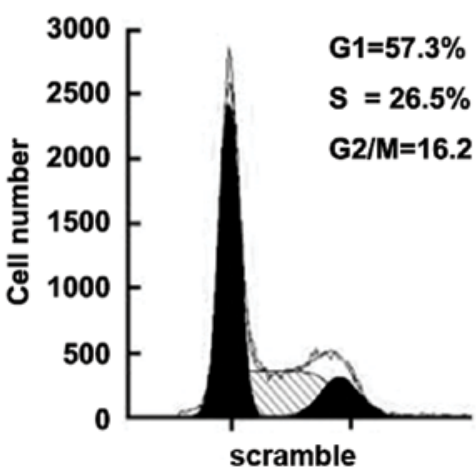

E
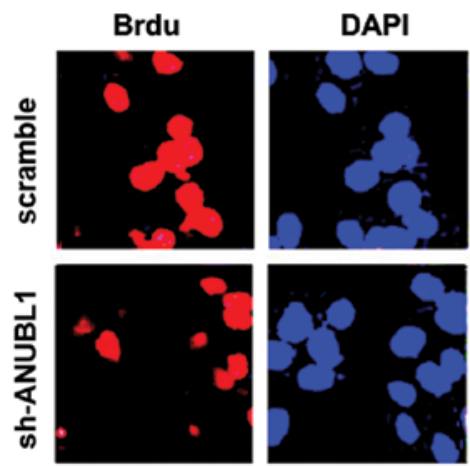

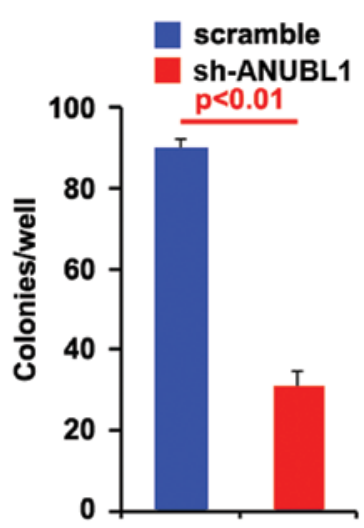

scramble sh-ANUBL1 $\mathrm{p}<0.01$

C
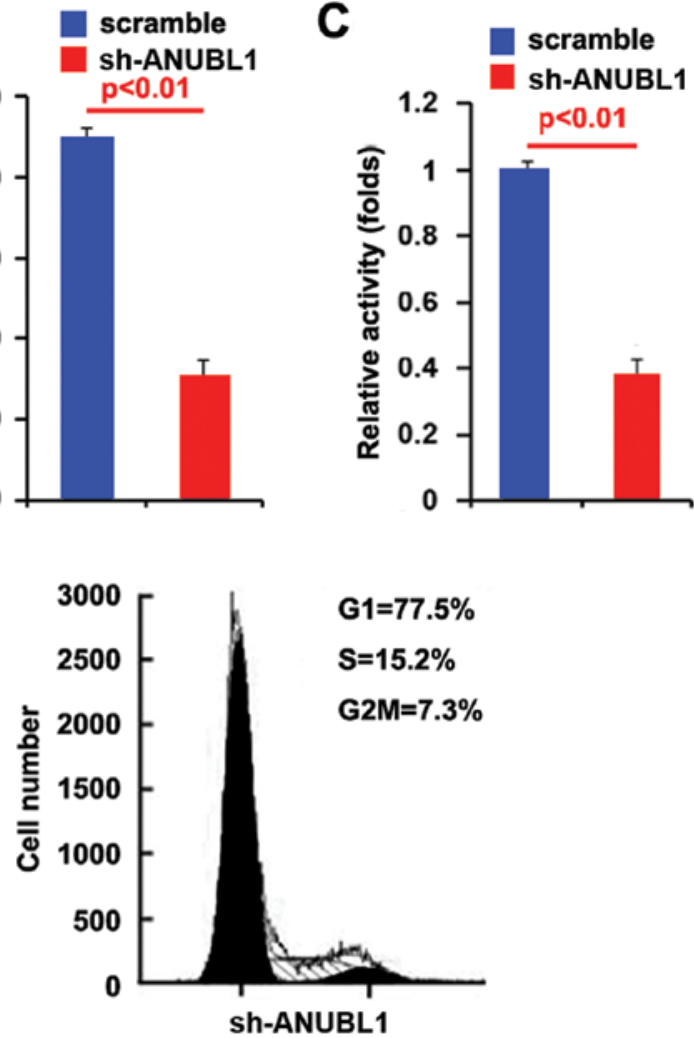

sh-ANUBL1

scramble

sh-ANUBL1 $p<0.01$
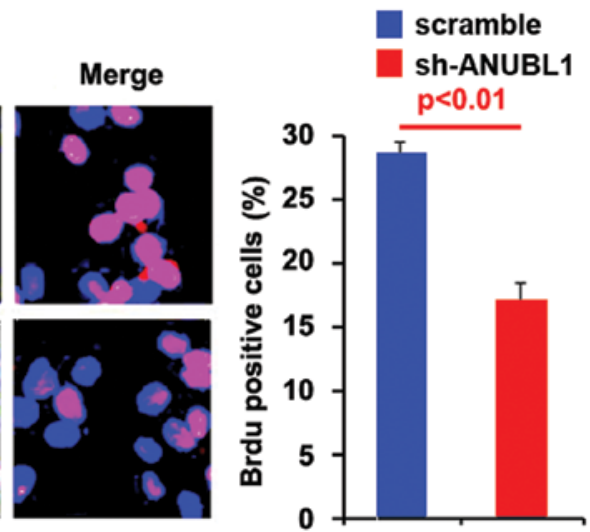

Figure 3. Sliencing ANUBL1 inhibits proliferation in gastric cancer SGC-7901 cells. (A) Western blot for ANUBL1 in SGC-7901 cells infected with sh-ANUBL1 or scramble. $\beta$-actin was a loading control $(\mathrm{n}=3)$. (B) Colony formation assay for SGC-7901 cells transfected with sh-ANUBL1 or scramble. Colonies with over 50 cells were counted. Representative micrographs (left) and quantification (right) of colonies after transfection with sh-ANUBL1 or scramble (n=3). (C) MTT assay for SGC-7901 cells transfected with sh-ANUBL1 or scramble (mock) (n=3). (D) Cell cycle analysis for SGC-7901 cells transfected with sh-ANUBL1 or scramble. Histograms of DNA contents obtained by FACS analysis are shown. The percentages of each cell cycle stages are shown in the inset of the histograms (n=3). (E) Brdu incorporation assay for SGC-7901 cells. Representative micrographs (left) and quantification (right) of BrdU incorporating cells with sh-ANUBL1 or scramble transfection $(n=3)$.

we next studied the mechanisms promoting ANUBL1 expression in the disease. MicroRNAs (miRNAs) are a new class of small ( 22 nucleotide) noncoding RNAs negatively regulating protein-coding gene expression by targeting mRNA degradation or translation inhibition (16). Downregulation of specific miRNA can contribute to oncogene overexpression (12). Thus we reasoned ANUBL1 was upregulated by defect of a specific miRNA.

As further confirmation, on the one hand, we employed four commonly used prediction algorithms - miRanda (http:// www.microrna.org/), TargetScan (http://www.targetscan.org), Pic Tar (http://pictar.mdc-berlin.de/) and miRDB (http://mirdb.
org/miRDB/index.html) to analyze 3'UTR of ANUBL1. All the four algorithms predicted that miR-182 could target 3'UTR of ANUBL1 (Fig. 4A) and the predicted target is shown in Fig. 4B.

To study biology function of miR-182, we tested whether miR-182 expression could be increased by pre-miR-182 in SGC-7901 cells. Real-time PCR was performed to detect miR-182 expression in the cells transfected with pre-miR-182 and the results showed that pre-miR-182 could significantly upregulate miR-182 expression (Fig. 4C).

In order to identify that ANUBL1 could be downregulated by miR-182, we performed immunofluorescence analyses 
A

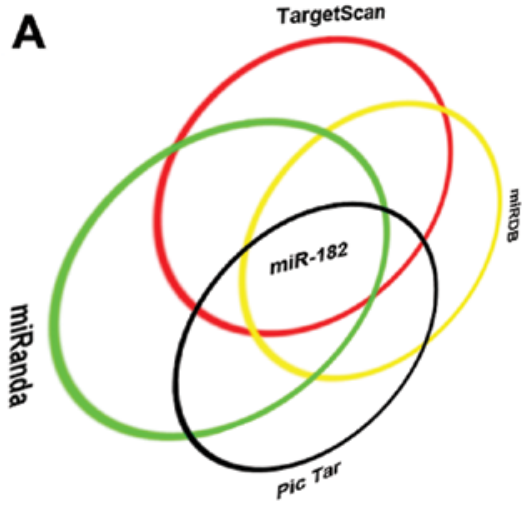

B

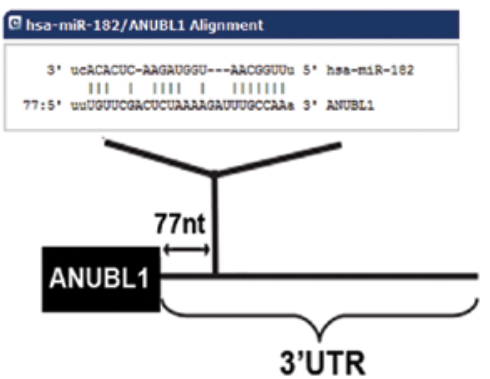

C

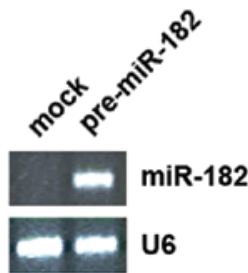

D
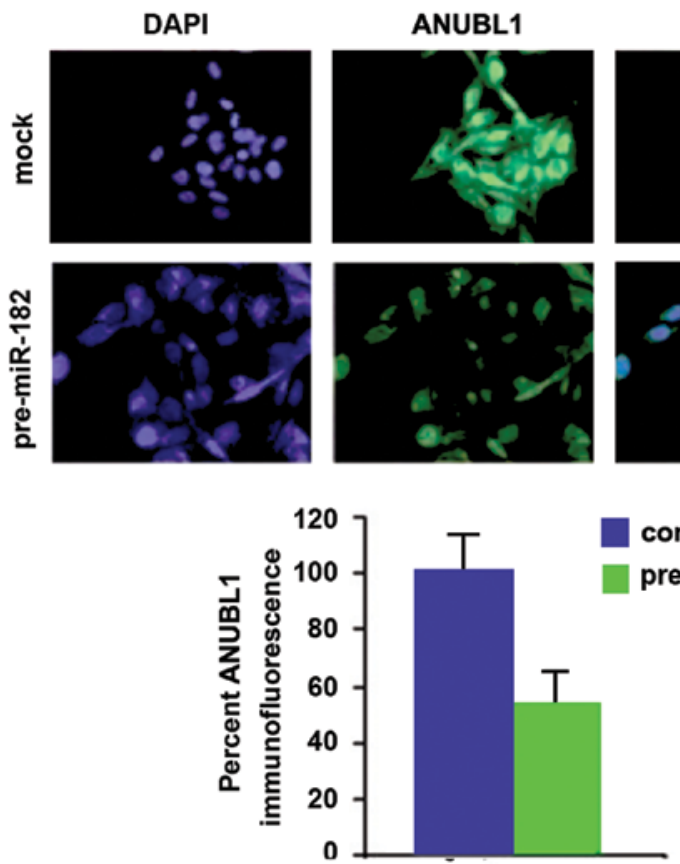

G

$$
\begin{gathered}
\text { 3' UCACACUCAAGAUGGUAACGgUUU 5' miR-182 } \\
77 \text { 5', UUUGUUCGACUCUAAAAGAUUU'I' I'́ } \\
77 \text { 5' UUUGUUCGACUCUAAAAGAUUUAAAAAA 3' ANUBL1-wt }
\end{gathered}
$$

E

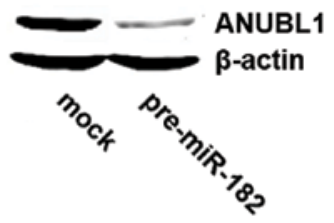

\section{F}

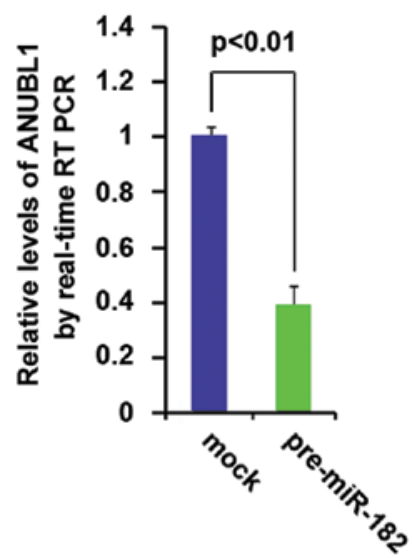

H

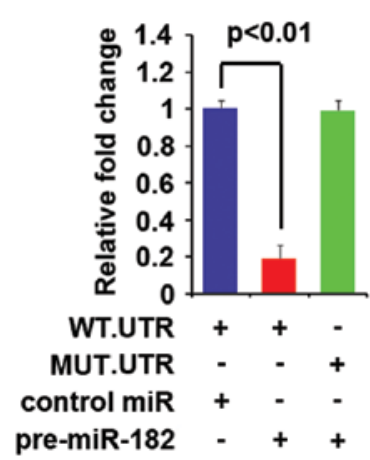

Figure 4. ANUBL1 is a target of miR-182 in gastric cancer SGC-7901 cells. (A) Diagram showing that ANUBL1 was a target gene of miR-182, predicted by miRanda, TargetScan, miRDB and PicTar. (B) Schematic for miR-182 and 3'UTR of ANUBL1. (C) Detection of miR-182 by RT-PCR in SGC-7901 cells transfected with pre-miR-182 or control miR (mock) (n=3). (D) Immunofluorescence analyses of SGC-7901 cells transfected with pre-miR-182 or control miR (mock). Upper panel shows microscopic images of immunofluorescence staining of one representative experiment (x100 magnification). Lower panel shows graphic presentation of mean fluorescence intensities of three independent experiments. (E) Western blot for ANUBL1 in SGC-7901 cells. SGC-7901 cells were transfected with pre-miR-182 or control-miR (mock). $\beta$-actin was a loading control ( $\mathrm{n}=3$ ). (F) Real-time PCR for ANUBL1 in SGC-7901 cells. SGC7901 cells were transfected with pre-miR-182 or control-miR (mock). U6 was a loading control (n=3). (G) Diagram of ANUBL1-3'UTR-containing reporter constructs. MUT: contains 3-base-mutation at the miR-182-target region, abolishing its binding. (H) Reporter assay, with cotransfection of 500 ng WT-or MUT-reporter and $50 \mathrm{nM}$ control-miR, or pre-miR-182 as indicated. At $36 \mathrm{~h}$ following transfection, cells were harvested for luciferase reporter assay ( $\mathrm{n}=3$ ).

and western blot to study whether miR-182 could affect ANUBL1 protein expression. Immunofluorescence analyses showed that ANUBL1 was significantly downregulated in SGC-7901 cells transfected with pre-miR-182 (Fig. 4D). 

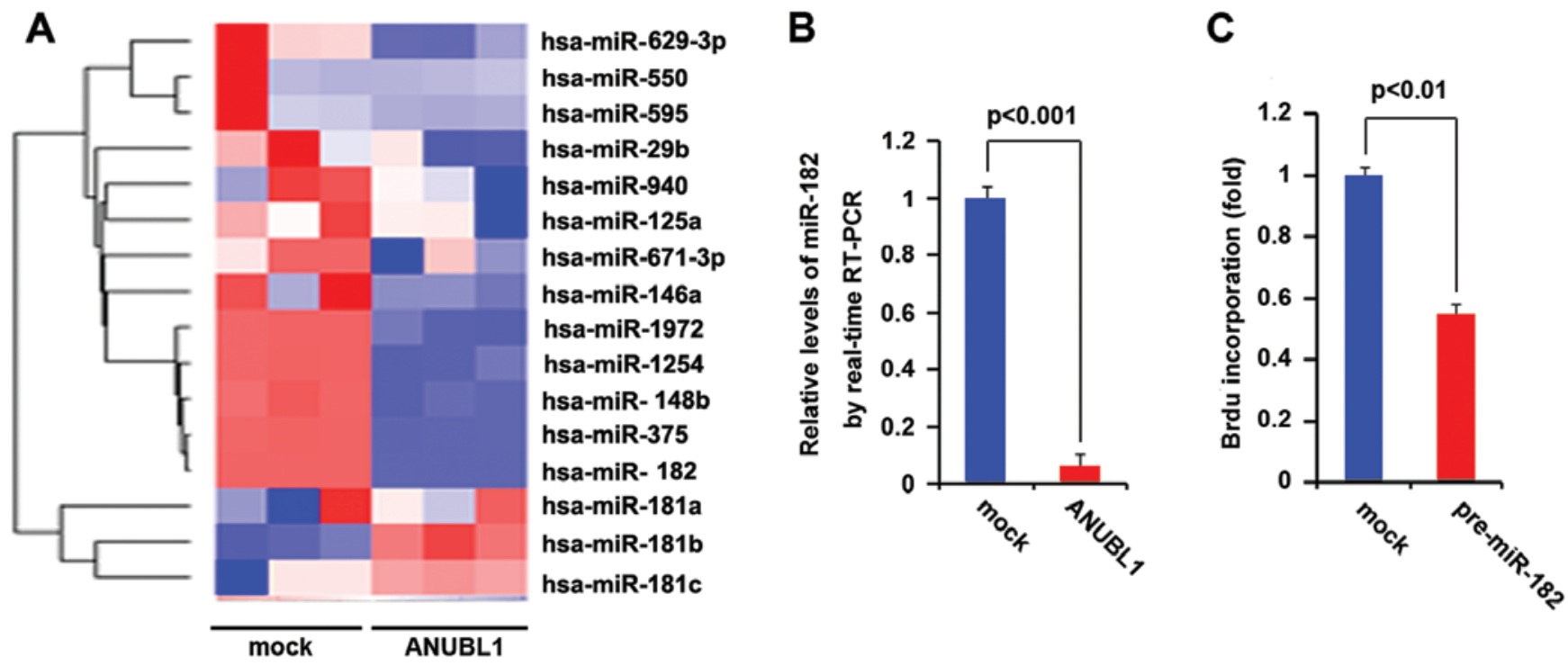

Figure 5. ANUBL1 regulates proliferation-associated microRNA expression. (A) Microarray analysis of SGC-7901 cells transfected with ANUBL1 expressing plasmids or empty vector (mock) was performed after transfection. Genes with similar expression profiles were grouped together using hierarchical clustering and the resulting genes tree is shown. Color bar indicates magnitude of gene regulation, with blue and red indicating repression and stimulation, respectively $(n=3)$. (B) Real-time PCR for miR-182 in SGC-7901 cells. U6 was a loading control ( $n=3)$. (C) Brdu incorporation analysis for SGC-7901 cells. SGC-7901 cells were transfected with pre-miR-182 or control-miR (mock) $(\mathrm{n}=3)$.

Consistent with immunofluorescence analyses, the results of western blot showed that ANUBL1 protein was significantly downregulated by miR-182 in SGC-7901 cells (Fig. 4E). We also performed real-time PCR to detect ANUBL1 mRNA in the cells transfected with pre-miR-182. The result showed that miR-182 indeed downregulated ANUBL1 mRNA expression in SGC-7901 cells (Fig. 4F).

To further demonstrate the direct regulation of ANUBL1 by miR-182, we used luciferase reporters with the targeting sequences of wild-type (ANUBL1-WT-luc) and mutated ANUBL1 3'UTRs (ANUBL1-MUT-luc) (Fig. 4G). To detect whether miR-182 targets 3'UTR of ANUBL1, luciferase assay was performed. Our results showed that miR-182 inhibited ANUBL1-WT-luc plasmids, but not ANUBL1-MUT-luc plasmids (Fig. 4H). All the results confirmed that miR-182 negatively regulates protein-coding gene ANUBL1 expression by targeting its 3'UTR.

ANUBL1 regulates proliferation-associated microRNA expression. Oncogenes or tumor suppressor genes can play important roles through regulating miRNAs in gastric cancer (17-19). Having demonstrated that ANUBL1, as an oncogene, promoted proliferation of SGC-7901 cells, we reasoned that ANUBL1 also could regulate proliferationassociated microRNA expression. In an attempt to identify that ANUBL1 contributed to aberrant regulation of miRNA expression in SGC-7901 cells, we performed miRNA profiling in SGC-7901 cells transfected with ANUBL1 expressing plasmids or empty vector. RNAs isolated from the cells were hybridized to a custom miRNA microarray platform. Following three hybridization cycles, quantification, and normalization, a dozen miRNAs, were changed in the cells transfected with ANUBL1 expressing plasmids. We were interested in miR-148b, miR-375 and miR-182, because they could suppress proliferation of gastric cancer cells $(12,20,21)$ and they were downregulated in the cells transfected with ANUBL1 expressing plasmids (Fig. 5A). We also performed real-time PCR to identify the miR-182 expression in the cells transfected with ANUBL1 expressing plasmids, and the results showed that ANUBL1 significantly inhibited the expression of miR-182 (Fig. 5B). We performed Brdu incorporation assay in SGC-7901 cells transfected with pre-miR-182. Consistent with previous studies (12), the results showed that miR-182 could inhibit DNA synthesis in SGC-7901 cells (Fig. 5C).

Introduction of ANUBL1 cDNA lacking predicted sites of 3'-UTR abrogates miR-182 cellular function. Since miR-182 directly degraded ANUBL1 through targeting its 3'UTR, we reasoned that ectopic expression of ANUBL1 by transfection of the cDNA that did not contain the predicted target of 3'UTR (in this study, the ANUBL1 expression plasmids did not contain the predicted position in its $3^{\prime}$ UTR) escapes the regulation of miR-182 and thus attenuate or decrease the function of miR-182. To this end, we transfected ANUBL1 expressing plasmids or empty vector (pcDNA3.1) into control miR or pre-miR-182 treated-SGC-7901 cells. Immunoblotting analysis revealed that transfection of ANUBL1 expressing plasmids attenuated the effect of miR-182 on ANUBL1 protein (Fig. 6A).

Since overexpression of miR-182 in SGC-7901 cells inhibited proliferation, in order to identify whether ANUBL1 could abrogate or attentuate the roles of miR-182 on proliferation, control miR or pre-miR-182-treated SGC-7901 cells were transfected with either ANUBL1 expressing plasmids or empty vector (pcDNA3.1), we performed MTT and Brdu incorporation assay and found that pre-miR-182-treated SGC-7901 cells displayed $\sim 40 \%$ decrease in proliferation compared to control miR treated cells (Fig. 6B) and DNA synthesis (Fig. 6C). Restoration of ANUBL1 sufficed to 


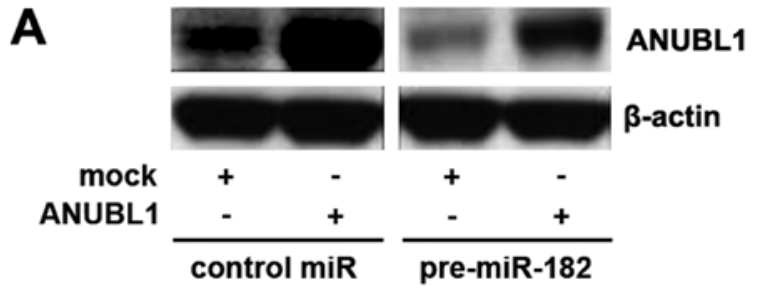

B
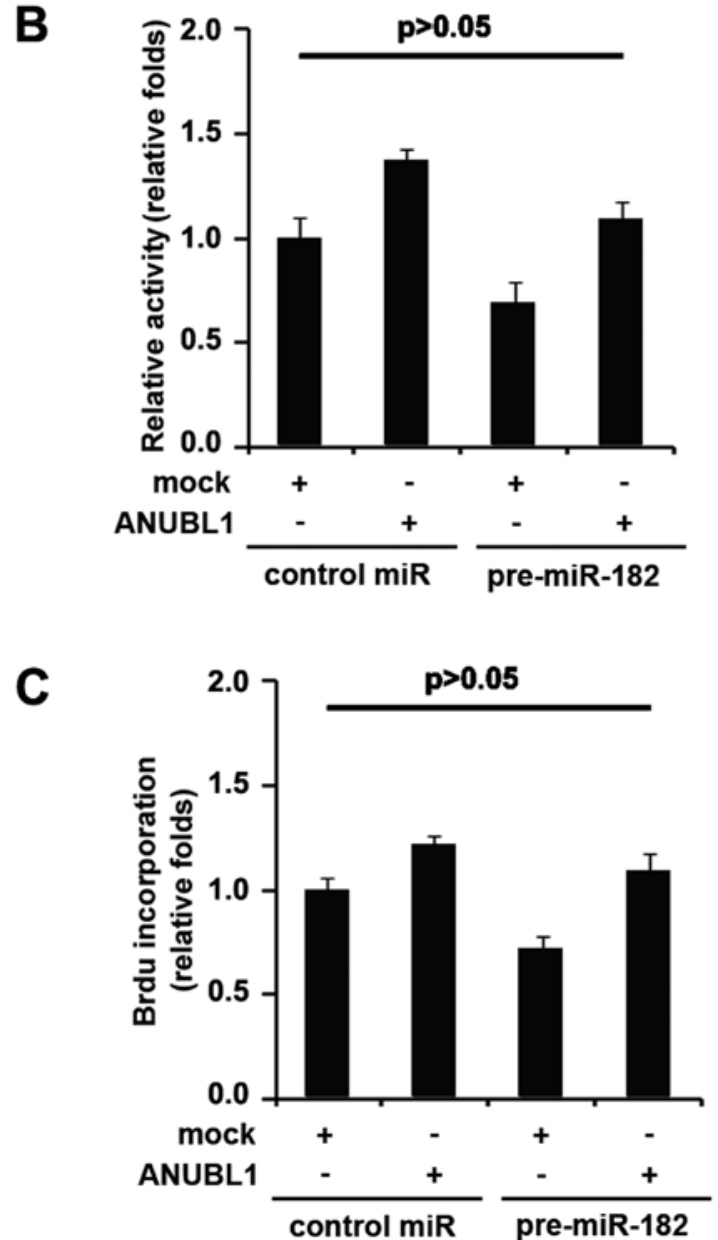

Figure 6. Introduction of ANUBL1 cDNA lacking predicted sites in 3'-UTR abrogates miR-182 cellular function in SGC-7901 cells. (A) Western blot for ANUBL1 in pre-miR-182 or control miR-treated-SGC-7901 cells transfected with ANUBL1 expressing plasmids or empty vector (mock). $\beta$-actin was a loading control $(n=3)$. (B) MTT assay for pre-miR-182 or control miRtreated-SGC-7901 cells transfected with ANUBL1 expressing plasmids or empty vector (mock) $(\mathrm{n}=3)$. (C) Brdu incorporation analysis for pre-miR-182 or control miR-treated-SGC-7901 cells transfected with ANUBL1 expressing plasmids or empty vector (mock) $(n=3)$.

reverse the loss of proliferation (Fig. 6B) and DNA synthesis (Fig. 6C) observed in pre-miR-182-treated cells. Thus, we concluded that miR-182 promoted proliferation via downregulating ANUBL1 expression.

\section{Discussion}

The interaction of multiple factors contributes to the development of gastric carcinogenesis, including regulatory changes in oncogenes and tumor suppressor genes $(3,4)$. The alterations of these genes are diverse. For example, the oncogenes $\mathrm{K}$-ras and c-met are significantly amplified and overexpressed in gastric cancer (22). Abnormal expression of CD44v is closely related to the invasiveness and metastasis of gastric cancer (23). As tumor suppressor genes, p53, APC and DCC show absent expression or lower expression levels in gastric cancer (24-26). More genes are still emerging as oncogenes or tumor suppressor genes in gastric cancer. In this study, we demonstrated that ANUBL1 expression was specifically upregulated in gastric cancer and it was positively associated with progression of gastric cancer. Moreover, as an oncogene, it can promote proliferation of gastric cancer SGC-7901 cells.

We also exploited the mechanism promoting ANUBL1 expression. We found that ANUBL1 is a target of miR-182. Because miR-182 is downregulated and can suppress cell growth in human gastric cancer (12), we reasoned that miR-182 inhibited proliferation by downregulating ANUBL1 expression. The fact that introduction of ANUBL1 cDNA lacking predicted sites in 3'-UTR abrogated miR-182 cellular function further confirmed the conclusion in the cells. Our results indicate that upregulation of ANUBL1 was associated with miR-182 low expression in gastric cancer. The elucidation of the mechanisms of miR-182 targeting ANUBL1 in gastric cancer helps us to further understand the mechanism of gastric cancer initiation and progression.

miR-182-mediated ANUBL1 regulation in gastric cancer demonstrated in this study has potential basic and clinical implications. On one hand, ANUBL1 could be a powerful oncogene by promoting proliferation and regulating relevant tumor suppressor genes in gastric cancer and pharmacological inhibition of ANUBL1 may represent a promising therapeutic strategy. On the other hand, miR-182 is a tumor suppressor gene and overexpression of ANUBL1 could further downregulate its expression. Further exploration of the role of ANUBL1 in cancer is required to confirm its clinical significance.

\section{Acknowledgments}

This study was supported by the Technology Development Foundation of Pudong District (PKJ2013-Y67), the Experimental Animal Special Purpose Foundation of Science and Technology Commission of Shanghai Municipality (13140902901). We thank Professor Zhen Zhan from Nanjing University of Chinese Medicine for constructive inputs and critical review of the manuscript.

\section{References}

1. Parkin DM, Bray F, Ferlay J, et al: Global cancer statistics 2002. CA Cancer J Clin 55: 74-108, 2005.

2. Khushalani N: Cancer of the esophagus and stomach. Mayo Clin Proc 83: 712-722, 2008.

3. Kim JM, Sohn HY, Yoon SY, et al: Identification of gastric cancer-related genes using a cDNA microarray containing novel expressed sequence tags expressed in gastric cancer cells. Clin Cancer Res 11: 473-482, 2005.

4. Chen X, Leung SY, Yuen ST, et al: Variation in gene expression patterns in human gastric cancers. Mol Biol Cell 14: 3208-3215, 2003.

5. Lee RC, Feinbaum RL and Ambros V: The C. elegans heterochronic gene lin-4 encodes small RNAs with antisense complementarity to lin-14. Cell 75: 843-854, 1993.

6. Pasquinelli AE, Reinhart BJ, Slack F, et al: Conservation of the sequence and temporal expression of let-7 heterochronic regulatory RNA. Nature 408: 86-89, 2000. 
7. Reinhart BJ, Slack FJ, Basson M, et al: The 21-nucleotide let-7 RNA regulates developmental timing in Caenorhabditis elegans. Nature 403: 901-906, 2000.

8. Li X, Zhang Y, Zhang Y, et al: Survival prediction of gastric cancer by a seven-microRNA signature. Gut 59: 579-585, 2009.

9. Ueda T, Volinia S, Okumura H, et al: Relation between microRNA expression and progression and prognosis of gastric cancer: a microRNA expression analysis. Lancet Oncol 11: 136-146, 2010.

10. Shimono Y, Zabala M, Cho RW, et al: Downregulation of miRNA-200c links breast cancer stem cells with normal stem cells. Cell 138: 592-603, 2009.

11. Sun Y, Fang R, Li C, et al: Hsa-miR-182 suppresses lung tumorigenesis through down regulation of RGS17 expression in vitro. Biochem Biophys Res Commun 396: 501-507, 2010.

12. Kong WQ, Bai R, Liu T, et al: MicroRNA-182 targets cAMPresponsive element-binding protein 1 and suppresses cell growth in human gastric adenocarcinoma. FEBS J 279: 1252-1260, 2012

13. Bacus SS, Zelnick CR, Plowman G, et al: Expression of the erbB-2 family of growth factor receptors and their ligands in breast cancers. Implication for tumor biology and clinical behavior. Am J Clin Pathol 102 (Suppl 1): S13-S24, 1994.

14. Liao XH, Lu DL, Wang N, et al: Estrogen receptor $\alpha$ mediates proliferation of breast cancer MCF-7 cells via a p21/PCNA/ E2F1-dependent pathway. FEBS J 281: 927-942, 2014.

15. Luo XG, Zou JN, Wang SZ, et al: Novobiocin decreases SMYD3 expression and inhibits the migration of MDA-MB-231 human breast cancer cells. IUBMB Life 62: 194-199, 2010.

16. Lu J, Getz G, Miska EA, et al: MicroRNA expression profiles classify human cancers. Nature 435: 834-838, 2005.

17. Li Z, Zhan W, Wang Z, et al: Inhibition of PRL-3 gene expression in gastric cancer cell line SGC7901 via micoRNA suppressed reduces peritoneal metastasis. Biochem Biophys Res Commun 348: 229-237, 2006.
18. Gao C, Zhan Z, Liu W, et al: Reduced microRNA-218 expression is associated with high nuclear factor kappa B activation in gastric cancer. Cancer 116: 41-49, 2010.

19. Gregory RI and Shiekhattar R: MicroRNA biogenesis and cancer. Cancer Res 65: 3509-3512, 2005.

20. Song YX, Yue ZY, Wang ZN, et al: MicroRNA-148b is frequently down-regulated in gastric cancer and acts as a tumor suppressor by inhibiting cell proliferation. Mol Cancer 10: 1, 2011.

21. Ding L, Xu Y, Zhang W, et al: miR-375 frequently downregulated in gastric cancer inhibits cell proliferation by targeting JAK2. Cell Res 20: 784-793, 2010.

22. Kaji M, Yonemura Y, Harada S, et al: Participation of c-met in the progression of human gastric cancers: anti-c-met oligonucleotides inhibit proliferation or invasiveness of gastric cancer cells. Cancer Gene Ther 3: 393-404, 1996.

23. Chen GY and Wang DR: The expression and clinical significance of CD44v in human gastric cancers. World J Gastroenterol 6: $125-127,2000$.

24. Tamura G, Kihana T, Nomura K, et al: Detection of frequent p53 gene mutations in primary gastric cancer by cell sorting and polymerase chain reaction single-strand conformation polymorphism analysis. Cancer Res 51: 3056-3058, 1991.

25. Horii A, Nakatsuru S, Miyoshi Y, et al: The APC gene, responsible for familial adenomatous polyposis, is mutated in human gastric cancer. Cancer Res 52: 3231-3233, 1992.

26. Uchino S, Tsuda H, Noguchi M, et al: Frequent loss of heterozygosity at the DCC locus in gastric cancer. Cancer Res 52: 3099-3102, 1992. 